\title{
Urea to Albumin Ratio Is an Independent Predictor of In-Hospital Mortality in Patients With Severe Pneumonia: A Retrospective Cohort Study
}

\author{
Yu Tian \\ Guangzhou Medical University \\ Yihao Li \\ Guangzhou Medical University \\ Jieru Chen ( $\nabla$ chenjieru77@163.com ) \\ Guangzhou Medical University \\ Zixin Jiang \\ Guangzhou Medical University
}

\section{Research}

Keywords: Severe pneumonia, Urea to albumin ratio, Intensive care unit, In-hospital mortality

Posted Date: May 13th, 2021

DOI: https://doi.org/10.21203/rs.3.rs-510525/v1

License: (c) (i) This work is licensed under a Creative Commons Attribution 4.0 International License. Read Full License 
1 Urea to albumin ratio is an independent predictor of in-

2 hospital mortality in patients with severe pneumonia: a

3 retrospective cohort study

$4 \quad$ Yu Tian ${ }^{1}$, Yihao $\mathrm{Li}^{2}$, Jieru Chen ${ }^{1 *}$, Zixin Jiang ${ }^{1 *}$

5 1ty2020guangzhou@163.com; ${ }^{2} \mathrm{dr}$ liyihao@163.com; *Correspondence: chenjieru77@163.com;

$6 \quad 147533981 @ q q . c o m$

7 Abstract

8 Background: Severe pneumonia (SP) is a major complication

9 of respiratory system disease that is associated with high mortality and morbidity. Our objective was to identify risk

11 factors predictive of SP patients and its mortality in intensive 12 care unit (ICU).

Methods: We conducted a single-center retrospective observational study involving 212 patients with SP in ICU from June 1st, 2016 to June 1st, 2020. The receiver operating characteristic (ROC) curve was constructed to assess the predictive significance of urea to albumin ratio (U/A). KaplanMeier survival curves were plotted with log-rank tests to compare survival of patients with different value of U/A. Multivariate COX regression models were used to calculate the adjusted hazard ratios (HR). Additionally, interaction analysis showed the association between U/A and in-hospital mortality 
1 was influenced by sex. Primary outcome was in-hospital

2 mortality.

3 Results: A total of 212 patients were enrolled in the analysis. In

4 the hospital, $101(47.6 \%)$ patients had died. ROC analysis

5 showed that the current cut-off of 0.2555 had a sensitivity of

$684.2 \%$ for in-hospital mortality (AUC $=0.63,95 \%$ CI: 0.55-0.70,

$7 \quad \mathrm{P}=0.001)$. The multivariate $\mathrm{COX}$ analysis showed that the

8 incidence of death was higher with the higher U/A group than

9 the lower group (HR: 2.234, 95\%CI: 1.146-4.356, P = 0.018). Besides, this pattern persisted in subgroup analyses considering sex. (HR: 9.380, 95\%CI: 2.248-39.138, $\mathrm{P}=0.002$ )

Conclusions: A high level of U/A is an independent risk factor for in-hospital mortality in patients with SP.

Keywords: Severe pneumonia, Urea to albumin ratio, Intensive care unit, In-hospital mortality

\section{Background}

Severe community-acquired pneumonia (SCAP) is a lifethreatening multifactorial clinical condition leading to a rapid deterioration of organ function associated with high mortality during hospitalization (ranging from $25 \%$ to more than $50 \%$ ) [1, 2]. SP in ICU must be treated promptly and effectively because 
1 of high mortality [3]. Therefore, severity evaluation is an

2 essential component of the initial assessment of these patients.

3 However, there is no consensus on the optimal evaluation

4 approach.

5 Risk factors for poor outcomes in patients with CAP include

6 higher blood urea nitrogen and lower albumin [3-7]. B/A levels

7 has also been reported to be associated with a high risk of 30-

8 day mortality in ventilator-associated pneumonia (VAP) patients

9 [8]. Mahmood Y et al. applied elevated urea and decreased albumin to COVID-19 pneumonia patients to predict the

11 admission to ICU [9]. Moreover, evidence is accumulating that a high blood urea nitrogen/albumin ratio (B/A) is relevant with critical illness [10]. However, there is no study on SP patients. higher risk of death than the lower group.

\section{Method}

Study design and Participants

We performed a retrospective and cohort study between June 1st, 2016, and June 1st, 2020, in the ICU of the Second 
1 Affiliated Hospital of Guangzhou Medical University after

2 obtaining institutional approval. Written informed consent was

3 approved by the retrospective nature.

4 Patients who were admitted to participating ICU were screened

5 and, if eligible, were included. We screened the patients 18

6 years of age or older who were admitted to the ICU for SP.

7 Patients were excluded for the reasons: (1) ICU duration $<24 \mathrm{~h}$;

8 (2) end-stage renal failure (on dialysis); (3) chronic liver

9 disease.

Definitions

To confirm reported clinical SP, the events were defined in a standardized approach with the use of criteria from the guidelines of SP in China (2016 version). Pneumonia was diagnosed when met one of the first four criteria and criteria 5:

(1) new cough or the ordinary respiratory disease worsened, with sputum and/or chest pain or not; (2) fever; (3) pulmonary moist rale and/or consolidation; (4) peripheral blood leucocyte count $>10 \times 10^{9} / \mathrm{L}$ or $<4 \times 10^{9} / \mathrm{L}$ with a nuclear shift to the left or not; (5) new chest radiographic infiltrate with pleural effusion or not and less possibility of alternative diagnoses. Pneumonia patients were diagnosed with SP when met one of the major 
1 criteria or three of the minor criteria. The major criteria include:

2 (1) invasive mechanical ventilation and (2) septic shock needing

3 vasopressor. The minor ones are: (1) respiratory rate $\geq 30$

4 breaths/min; (2) multilobar infiltrates; (3) $\mathrm{PaO} 2 / \mathrm{FiO} 2$ ratio $\leq$

5 250; (4) uremia (BUN level > 20mg/dL); (5)

6 confusion/disorientation; (6) leukopenia (WBC count <

$\left.74 \times 10^{9} / \mathrm{L}\right)$; (7) thrombocytopenia (platelet count $<100 \times 10^{9} / \mathrm{L}$ );

8 (8) hypothermia (core temperature $<36^{\circ} \mathrm{C}$ ); and (9) hypotension

9 requiring massive fluid resuscitation.

Data collection and outcome

Data included demographic data, hospital-acquired pneumonia (HAP), underlying disease, radiological findings,

treatment, clinical data, laboratory results, and clinical outcomes. Demographic data were age and gender. Underlying diseases included hypertension, diabetes mellitus, coronary heart disease (CHD), stroke, chronic obstructive pulmonary disease (COPD), and chronic kidney disease (CKD). Clinical and laboratory results contained mean arterial pressure (MAP), heart rate $(\mathrm{HR})$, respiratory rate (RR), acute physiology and chronic health evaluation II (APACHE II) score, alanine aminotransferase (ALT), aspartate aminotransferase (AST), 
1 creatinine, urea, albumin, white blood cell (WBC), neutrophil

2 count, lymphocyte count, monocyte count, platelet count, red

3 blood cell (RBC), hemoglobin, and hematocrit (HCT). Samples

4 of peripheral blood were stored by tubes with ethylenediamine

5 teraacetic acid. Primary outcome was in-hospital mortality.

$7 \quad$ Statistics analysis

8 The patients were divided into 2 groups according to the ROC

9 analysis cutoff values. The ROC curve was used to examine the predictive power, and the area under the ROC curve (AUC) was

11 represented the predictive power. Differences between two groups were tested using $t$ test, Mann-Whitney $U$ test or $C h i$ -

Square test where appropriate. The incidence of death was estimated by using the Kaplan-Meier method and compared with the log-rank test. The associations between U/A and the primary outcome were examined with use of multivariate $\mathrm{COX}$ models. Hazard ratios (HR), with the U/A $\leq 0.2555$ group used as the reference, were adjusted for sex and other significant univariate $(\mathrm{P}<0.05$ in univariate analysis). A formal test of interaction between U/A and sex was performed. The data missing under $5 \%$ were replaced by the mean or median. 
1 Statistical analyses were performed with the use of SPSS,

2 version 22.0 , and $\mathrm{P}<0.05$ was considered significant.

3

4 Results

5 Baseline Characters

6 From 1st June 2016 to 30th June 2020, a total of 227 patients

7 were screened in the ICU, and 212 patients were eventually

8 enrolled in the study (Figure 1). Patients' characteristics are presented in Table 1 . Of these 212 cases, the median age was $73.0(61.0,82.8), 0.8 \%$ of the patients were male, and $16.0 \%$ were hospital-acquired pneumonia (Table 1). Overall, the underlying disease of the patients were including hypertension, DM, CHD, stroke, COPD, CKD. The median Apache II score was $20.0(16.0,26.0)$ within the 24 hours after ICU admission. The radiological findings showed that $82.5 \%$ of the patients had bilateral pneumonia and $31.1 \%$ had pleural effusion. No significant difference in the radiological findings was observed ( $P=0.389, P=0.494$, respectively). During the follow-up, 101 $(47.6 \%)$ cases of death were recorded during hospitalization. Compare to the U/A $\leq 0.2555$ group, patients in the U/A > 0.2555 group required more continuous renal replacement treatment therapy (CRRT) and had higher in-hospital mortality 
1 as well as APACHE II score $(\mathrm{P}<0.001, \mathrm{P}<0.001, \mathrm{P}<0.001$,

2 respectively).

3

4 Risk Factors for Higher Mortality in SP Patients

5 Factors associated with higher in-hospital mortality are listed in

6 Table 2. All significant factors identified as predictors of in-

7 hospital mortality $(\mathrm{P}<0.05$ in the $\mathrm{COX}$ univariate regression

8 analysis and clinical concern(sex)) were used for the

9 multivariate analysis based on the COX proportional hazards regression. Multivariate $\mathrm{COX}$ analyses identified two prognostic

11 factors for in-hospital mortality, including vasopressor use and

CRRT $(\mathrm{P}=0.004, \mathrm{P}=0.041$, respectively). 
1 According to the cutoff value, the 211 SP patients were divided

2 into two groups. The Kaplan-Meier survival curves showed that

3 higher U/A group had a higher in-hospital mortality rate than

4 lower U/A group (Log-rank test chi-square 13.71, $\mathrm{P}<0.001$ ).

5 To elucidate the specific relationship between U/A and in-

6 hospital mortality, we used different models (Table 3, U/A $\leq$

$7 \quad 0.2555$ as the reference group). Using the multivariable COX

8 proportional hazards model, which adjusted vital factors

9 (univariate $\mathrm{COX}$ analysis, $\mathrm{P}<0.05$ and age), we discovered that in-hospital mortality was still significant higher in the group with U/A > 0.2555. In model 3 adjusted for age, sex, invasive mechanical ventilation, CRRT, vasopressor use, creatinine, alanine aminotransferase, and aspartate aminotransferase, the HR for in-hospital mortality was 2.234 (95\%CI: 1.146-4.356, P $=0.018)$.

Relationship between Mortality and U/A in the Sex Subgroups Results of interaction analysis between U/A and sex are given in Table 4. There was a significant interaction on in-hospitality mortality between them $(\beta=4.290, \mathrm{P}=0.004)$. Thus, a sexstratified analysis was conducted. In the female subgroup, COX analyses showed significant mortality increases with high value 
1 of U/A > 0.2555 (HR: 9.380, 95\%CI: 2.248-39.138, $\mathrm{P}=0.002$ ).

2 However, a pattern of increasing mortality risk with different

3 level of U/A was not observed in the male subgroup $(\mathrm{P}=0.112)$.

4

5 Discussion

6 Our analysis suggested that the first U/A after admitted to ICU is

7 an independent risk factor for in-hospital mortality in SP patients.

8 Interestingly, this study also demonstrated that U/A was an 9 independent predictor of in-hospital mortality in female subgroups, but not in males.

Urea and albumin are very easy and quick to get. Studies has shown that the higher urea and lower albumin indicated worse clinical outcome in CAP patients [3-7]. Motoi et al. revealed that the blood urea nitrogen/serum albumin (B/A) ratio performed well for predicting mortality and the severity of CAP [10]. Ding-Yun Feng et al.'s study showed that the B/A ratio was associated with poorer survival outcomes in 30-day ventilation acquired pneumonia (VAP) [11]. However, very few studies in the literature have evaluated whether U/A are predictive of worse outcomes in SP patients. Thus, according to the previous researches, we speculate that the U/A may be an important indicator of mortality in SP patients. The results of 
1 our study were consistent with this speculation. The present data

2 indicated U/A had a significant predictive value.

3 An earlier study calculated the optimal cutoff point of B/A

4 value for 30-day mortality using ROC curves in CAP patients.

5 The point was at 0.165 [9]. Seung Ryu et al. found that, in

6 aspiration pneumonia patients, the $\mathrm{AUC}$ for $\mathrm{B} / \mathrm{A}$ ratio was at

70.70 for predicting mortality within 28 days [12]. Our study

8 included both CAP and HAP participants in the ICU. In our

9 ROC curve analysis, we determined a cutoff value of 0.2555 for in-hospital mortality and the AUC was 0.63 . The risk of death was higher among the patients whose value of U/A was >

0.2555 than those whose U/A was $\leq 0.2555$ (HR: $2.234,95 \% \mathrm{CI}$ :

1.146-4.356, $\mathrm{P}=0.018)$. Although the AUC of U/A was not so good, it is easy and quick to use, giving more information to identify the high-risk group.

However, the underlying mechanism has remained unclear. Urea is a marker associated with systemic disease. Although urea is not a direct mark of infection, it can be a risk factor because high value leads to high susceptibility to infection. Some previous studies suggested that urea affects the prognosis of critical patients regardless of the creatine level $[13,14]$. In these prediction model, urea is a significant risk factor for 
1 pneumonia. Moreover, urea is an indirect marker of a metabolic

2 systemic pathway [15]. In pneumonia patients, elevations of

3 serum urea are indicators of protein catabolism. Water

4 deficiency appears to be common in pneumonia patients. In the

5 process of dehydration, the concentration of urea increased.

6 Meanwhile, the effect of increased urea reabsorption in the

7 kidney causes high urea concentration [12]. Additionally, urea

8 level is regarded as a predictive marker reflecting the

9 cumulative effects of hemodynamic damage, which is essential

10 in critical illness.

11 Serum albumin plays a significant role in maintaining physiological homeostasis, including keeping a colloid osmotic pressure [16]. On the other hand, hypoalbuminemia can result in the pulmonary edema due to decreased colloid osmotic pressure which can result in mortality[17]. Xue et al. suggested that hypoalbuminemia in the early stage had a high incidence of infection and mortality [18]. At the same time, pneumonia is an inflammation with high catabolism condition. Systemic inflammatory response can decrease serum albumin levels [16]. Obviously, hypoalbuminemia is often observed in malnutrition patients, resulting in worse outcomes. It is interesting to note that earlier studies mostly focused on CAP showed that non- 
1 survivors have significantly lower urea and higher albumin than

2 those of survivors. The study reported before demonstrated that

3 urea to albumin ratio is an independent marker of the severity of

4 CAP and mortality [16]. Our findings are consistent with

5 previous conclusions.

Another notable finding was the independent effect of increased U/A on the elevated risk associated with in-hospital mortality in female SP patients. To the authors' knowledge, this research may be the first time to revealed the association between U/A and in-hospital mortality in the female. Our study found an interaction between U/A and sex. In subgroup analyses by sex, U/A was still an independent risk factor for in-hospital mortality in female. Nevertheless, the same pattern was not observed in the male subgroups.

A previous study reported that males had a higher fractional synthesis rate of albumin than females regardless of age and protein intake. Male had higher albumin concentration than female [19]. Therefore, the increase of U/A in critical illness was not so obvious. Gary Weaving et al demonstrated that albumin value in females decreased more quickly [20]. This is owing to the different values of parameters between males and females. Our results are consistent with the previous studies. 
1 Further studies are needed to examine why U/A is associated

2 with mortality in female patients.

3 Our research has some limitations. First, the retrospective

4 design of the study could lead to residual confounders bias. It

5 might be insufficient to draw the same conclusion in other

6 population. Second, the samples were small, so the predictive

7 value of the U/A needs to be further validated in other

8 observational studies. Third, the AUC of the ROC curve was

9 0.63. It showed that the U/A had moderate predictive function on the prognosis of SP. Fourth, in the present study, we investigated first time U/A value in patients with SP who were admitted to the ICU for first time. The relationship between the variation of U/A level and the primary outcome remains uncertain. Finally, our analysis relies on in-hospital mortality and just reflets the time within hospital.

In conclusion, our study demonstrated that the U/A is an independent risk factor for in-hospital mortality.

\section{Abbreviations}

SP: Severe pneumonia; ICU: Intensive care unit; ROC curve: Receiver operating characteristic curve; U/A: Urea to albumin ratio; HR: Hazard ratios; SCAP: Severe community-acquired pneumonia; VAP: Ventilator-associated pneumonia; HAP: Hospital acquired pneumonia; CHD: 
1 Coronary heart disease; COPD: Chronic obstructive pulmonary disease; CKD: Chronic kidney

2 disease; CRRT: Continuous renal replacement therapy; MAP: Mean arterial pressure; APACHE:

3 Acute physiology and chronic health evaluation; ALT: Alanine aminotransferase; AST: Aspartate

4 aminotransferase; WBC: White blood cell; RBC: Red blood cell; HCT: Hematocrit; LOS: Length

5 of stay

6

$7 \quad$ Acknowledgements

$8 \quad$ None applicable.

9

10 Authors' contributions

11 Yu Tian and Yihao Li contributed equally to this work.

12

$13 \quad$ Funding

14 Not applicable.

15

16 Availability of data and materials

17 The data used to support the findings of this study are included within the article. 


\section{Declarations}

\section{Ethical Approval}

3 All procedures performed in studies involving human participants were in accordance with the

4 ethical standards of the institutional and/or national research committee at which the studies were

5 conducted and with the 1964 Helsinki Declaration and its later amendments or comparable ethical

6 standards.

7

\section{Consent for publication}

$9 \quad$ Not applicable.

10

11 Competing interests

12 The authors declare that they have no conflict of interest.

\section{Author details}

$15{ }^{1}$ Department of Intensive Care Unit, The Second Affiliated Hospital, Guangzhou Medical

16 University, Guangzhou 510000, PR China. ${ }^{2}$ Department of Anesthesiology, The Second

17 Affiliated Hospital, Guangzhou Medical University, Guangzhou 510000, PR China. 
2 Reference

3 1. Cillóniz C, Dominedò C, Garcia-Vidal C, Torres A. Community-acquired pneumonia as an emergency condition. Current opinion in critical care; 2018;24(6):531-539.

2. Montull B, Menéndez R, Torres A, Méndez R. Predictors of severe sepsis among patients hospitalized for community-acquired pneumonia. MLO: medical laboratory observer; 2016;48(3):8, 10-12. https://core.ac.uk/reader/154764369?utm_source=linkout

3. Bodmann KF. Current guidelines for the treatment of severe pneumonia and sepsis. Chemotherapy. 2005;51(5):227-233. https://doi.org/10.1159/000087452

4. Lim WS, van der Eerden MM, Laing R, Boersma WG, Karalus N, Town GI, Lewis SA, Macfarlane JT. Defining community acquired pneumonia severity on presentation to hospital: an international derivation and validation study. Thorax. 2003;58(5):377-382.

5. Farr BM, Sloman AJ, Fisch MJ. Predicting death in patients hospitalized for

7. Potgieter PD, Hammond JM. The intensive care management, mortality and prognostic

8. Lee JH, Kim J, Kim K, Jo YH, Rhee J, Kim TY, Na SH, Hwang SS. Albumin and C-

9. Feng DY, Zhou YQ, Zou XL, Zhou M, Yang HL, Chen XX, Zhang TT. Elevated Blood 
Mortality of Patients with Hospital-Acquired Pneumonia. The Canadian journal of infectious diseases \& medical microbiology. 2019;2019:1547405. https://doi.org/10.1155/2019/1547405

0. Hachim MY, Hachim IY, Naeem KB, Hannawi H, Salmi IA, Hannawi S. D-dimer, Troponin, and Urea Level at Presentation With COVID-19 can Predict ICU Admission: A Single Centered Study. Frontiers in medicine. 2020;7:585003. https://doi.org/10.3389/fmed.2020.585003

11. Ugajin M, Yamaki K, Hirasawa N, Yagi T. Predictive values of semi-quantitative procalcitonin test and common biomarkers for the clinical outcomes of communityacquired pneumonia. Respiratory care. 2014;59(4):564-573. https://doi.org/10.4187/respcare.02807

12. Ryu S, Oh SK, Cho SU, You Y, Park JS, Min JH, Jeong W, Cho YC, Ahn HJ, Kang C. Utility of the blood urea nitrogen to serum albumin ratio as a prognostic factor of mortality in aspiration pneumonia patients. The American journal of emergency medicine. 2020. https://doi.org/10.1016/j.ajem.2020.02.045

13. Fine MJ, Auble TE, Yealy DM, Hanusa BH, Weissfeld LA, Singer DE, Coley CM, Marrie TJ, Kapoor WN. A prediction rule to identify low-risk patients with communityacquired pneumonia. $\quad N \quad$ Engl $J \quad$ Med. 1997;336(4):243-250. https://doi.org/10.1056/nejm199701233360402

14. Aronson D, Mittleman MA, Burger AJ. Elevated blood urea nitrogen level as a predictor of mortality in patients admitted for decompensated heart failure. The American journal of medicine. 2004;116(7):466-473. https://doi.org/10.1016/j.amjmed.2003.11.014

15. Oh DH, Kim YC, Kim EJ, Jung IY, Jeong SJ, Kim SY, Park MS, Kim A, Lee JG, Paik HC. Multidrug-resistant Acinetobacter baumannii infection in lung transplant recipients: risk factors and prognosis. Infectious diseases (London, England). 2019;51(7):493-501. https://doi.org/10.1080/23744235.2018.1556400

16. Ugajin M, Yamaki K, Iwamura N, Yagi T, Asano T. Blood urea nitrogen to serum albumin ratio independently predicts mortality and severity of community-acquired pneumonia. International journal of general medicine. 2012;5:583-589. https://doi.org/10.2147/ijgm.s33628 
1 17. Horwich TB, Kalantar-Zadeh K, MacLellan RW, Fonarow GC. Albumin levels predict 5 17 survival in patients with systolic heart failure. American heart journal. 2008;155(5):883-889. https://doi.org/10.1016/j.ahj.2007.11.043

18. Xue P, Huang ZW, Li YH, Guo J, Wang ZC, Zhao JL, You Z. Clinical study on severe acute pancreatitis associated with hypoalbuminemia in early stage. Journal of Chinese integrative medicine. 2005;3(6):443-445.

19. Thalacker-Mercer AE, Johnson CA, Yarasheski KE, Carnell NS, Campbell WW. Nutrient ingestion, protein intake, and sex, but not age, affect the albumin synthesis rate in humans. The Journal of nutrition. 2007;137(7):1734-1740.

20. Weaving G, Batstone GF, Jones RG. Age and sex variation in serum albumin concentration: an observational study. Annals of clinical biochemistry. 2016;53(Pt 1):106-111. https://doi.org/10.1177/0004563215593561 4 16 
1 Table 1: Comparison of baseline characteristics.

\begin{tabular}{|c|c|c|c|c|}
\hline Variables & Total $(\mathrm{n}=212)$ & $\mathrm{U} / \mathrm{A} \leq 0.2555(\mathrm{n}=58)$ & $\mathrm{U} / \mathrm{A}>0.2555(\mathrm{n}=154)$ & P-value \\
\hline \multicolumn{5}{|c|}{ Demographic data } \\
\hline Age(years) & $73.0(61.0,82.8)$ & $66.0(53.8,78.3)$ & $78.5(63.0,84.0)$ & 0.001 \\
\hline Sex(male) & $150,70.8 \%$ & $34,58.6 \%$ & $116,75.3 \%$ & 0.017 \\
\hline HAP & $34,16.0 \%$ & $8,13.8 \%$ & $26,16.9 \%$ & 0.585 \\
\hline
\end{tabular}

Underlying diseases

Hypertension

$119,56.1 \%$

$21,36.2 \%$

$98,63.6 \%$

$<0.001$

Diabetes mellitus

$53,25.0 \%$

$10,17.2 \%$

43, $27.9 \%$

0.109

CHD

$25,11.8 \%$

4, $6.9 \%$

$21,13.6 \%$

0.175

Stroke

$44,20.8 \%$

$8,13.8 \%$

$36,23.4 \%$

0.125

COPD

$27,12.7 \%$

4, $6.9 \%$

$23,14.9 \%$

0.118

CKD

$27,12.7 \%$

$1,1.7 \%$

$26,16.9 \%$

0.003

Radiological findings

Bilateral pneumonia

$175,82.5 \%$

$50,86.2 \%$

$125,81.2 \%$

0.389

Pleural effusion

$66,31.1 \%$

$16,27.6 \%$

$50,32.5 \%$

0.494 
Treatment

Invasive Mechanical use

$109,51.4 \%$

$34,58.6 \%$

$75,48.7 \%$

0.294

Vasopressor Use

$73,34.4 \%$

$14,24.1 \%$

$59,38.3 \%$

0.053

CRRT

$73,34.4 \%$

7, $12.1 \%$

$66,42.9 \%$

$<0.001$

Clinical data

MAP

$83.0(69.5,103.0)$

$85.0(74.6,108.5)$

$83.0(66.9,101.3)$

0.153

Heart Rate

$109.9(83.8,136.0)$

$104.5(84.4,124.6)$

$111.9(84.1,139.7)$

0.035

Respiratory rate

$27.0(22.0,34.8)$

$25.5(20.0,33.3)$

$27.0(22.0,35.0)$

0.161

APACHE II score

$20.0(16.0,26.0)$

$16.5(12.0,20.0)$

$22.0(17.0,27.3)$

$<0.001$

Laboratory results

ALT

$34.5(21.0,65.8)$

$33.0(21.0,51.3)$

$34.8(21.0,76.8)$

0.265

AST

$44.5(27.0,82.0)$

$42.5(24.5,60.8)$

$44.5(28.0,97.0)$

0.054

Creatinine

$114.9(72.8,191.4)$

$65.2(51.1,85.6)$

$140.4(98.0,239.0)$

$<0.001$

Urea

$11.8(6.9,19.1)$

$5.5(4.2,6.5)$

$15.4(11.1,21.6)$

$<0.001$

Albumin

$29.0(24.1,33.9)$

$30.8(26.5,35.1)$

$28.3(23.3,33.3)$

0.001 


\begin{tabular}{|c|c|c|c|c|}
\hline WBC & $11.7(8.0,16.0)$ & $12.9(9.9,16.8)$ & $11.2(6.8,15.5)$ & 0.035 \\
\hline Neutrophil count & $10.0(6.3,13.9)$ & $11.0(8.4,14.6)$ & $9.7(5.4,13.8)$ & 0.072 \\
\hline Lymphocyte count & $0.6(0.3,1.0)$ & $0.8(0.4,1.3)$ & $0.6(0.3,0.9)$ & 0.006 \\
\hline Monocyte count & $0.5(0.2,0.8)$ & $0.6(0.3,1.1)$ & $0.5(0.2,0.8)$ & 0.008 \\
\hline Platelet count & $205.0(117.3,280.5)$ & $234.5(176.8,322.0)$ & $186.5(98.3,256.0)$ & $<0.001$ \\
\hline $\mathrm{RBC}$ & $3.6(2.6,4.6)$ & $4.0(3.1,4.9)$ & $3.4(2.4,4.4)$ & $<0.001$ \\
\hline Hemoglobin & $102.9(73.8,132.0)$ & $112.9(89.2,136.6)$ & $99.1(69.0,129.2)$ & 0.001 \\
\hline НCT & $31.3(22.7,39.9)$ & $33.9(26.6,41.2)$ & $30.3(21.4,39.2)$ & 0.004 \\
\hline Clinical Outcomes & $12.0(6.3,21.0)$ & & & \\
\hline ICU LOS & $21.0(11.3,32.0)$ & $14.0(9.0,24.3)$ & $11.0(5.0,19.3)$ & 0.020 \\
\hline Hospital LOS & $101,47.6 \%$ & $25.0(15.5,36.3)$ & $18.0(10.0,32.0)$ & 0.040 \\
\hline In-hospital death & & $16,27.6 \%$ & $85,55,2 \%$ & $<0.001$ \\
\hline
\end{tabular}

1 Data are mean \pm standard or medians (25th-75th percentile) or number and percentage.

2 U/A: urea to albumin ratio; HAP: hospital acquired pneumonia; CHD: coronary heart disease;

3 COPD: chronic obstructive pulmonary disease; CKD: chronic kidney disease; CRRT: continuous

4 renal replacement therapy; MAP: mean arterial pressure; APACHE: acute physiology and chronic 

blood cell; RBC: red blood cell; HCT: hematocrit; LOS: length of stay.

3

4

8

9

10

11

12

13

14

15

16 
1 Table 2: Independent predictors of in-hospital mortality by univariate and multivariate COX regression analysis.

\begin{tabular}{|c|c|c|}
\hline Factors & $\mathrm{HR} \square 95 \% \mathrm{CI} \square$ & $P$ \\
\hline \multicolumn{3}{|l|}{ Univariate cox analysis } \\
\hline Age & $1.013(1.001-1.026)$ & 0.029 \\
\hline Mechanical ventilation & $0.796(0.641-0.990)$ & 0.040 \\
\hline Vasopressor use & $2.407(1.619-3.578)$ & $<0.001$ \\
\hline CRRT & $2.402(1.607-3.592)$ & $<0.001$ \\
\hline ALT & $1.001(1.000-1.001)$ & 0.006 \\
\hline Albumin & $0.947(0.908-0.988)$ & 0.011 \\
\hline Urea & $1.020(1.003-1.037)$ & 0.024 \\
\hline AST & $1.001(1.000-1.001)$ & $<0.001$ \\
\hline Creatinine & $1.002(1.000-1.003)$ & 0.016 \\
\hline \multicolumn{3}{|l|}{ Multivariate cox analysis } \\
\hline Vasopressor use & $1.888(1.226-2.907)$ & 0.004 \\
\hline CRRT & $1.679(1.020-2.762)$ & 0.041 \\
\hline
\end{tabular}

3 Covariates included in multivariate analysis: age, sex, mechanical ventilation, vasopressor use,

4 CRRT, ALT, albumin, urea, AST, creatinine.

5 CRRT: continuous renal replacement treatment; ALT: alanine aminotransferase; AST: aspartate 6 aminotransferase; HR: hazard ratio; CI: confidence interval.

7 
1 Table 3: Relationship between U/A level and in-hospital mortality.

\begin{tabular}{lll}
\hline In-hospital mortality & $\mathbf{U} / \mathbf{A}>\mathbf{0 . 2 5 5 5}$ group & \\
\cline { 2 - 3 } & $\mathrm{HR}(95 \% \mathrm{CI})$ & $\mathrm{P}$ \\
\hline Unadjusted & $2.788(1.577-4.929)$ & $<0.001$ \\
Model 1 & $2.724(1.499-4.949)$ & 0.001 \\
Model 2 & $2.080(1.100-3.934)$ & 0.024 \\
Model 3 & $2.234(1.146-4.356)$ & 0.018 \\
\hline
\end{tabular}

Reference group is U/A $\leq 0.2555$ group.

$3 \quad$ Model 1: age and sex

4 Model 2: Model1 plus treatment (mechanical ventilation, CRRT, vasopressor use)

$5 \quad$ Model 3: Model2 plus and laboratory test $\square$ Creatinine $\square$ ALT $\square$ A ST $\square$

6 CRRT: continuous renal replacement treatment; ALT: alanine aminotransferase; AST: aspartate

7 aminotransferase; HR: hazard ratio; CI: confidence interval.

8

9

10

11

12

13

14 
1 Table 4: Relationship between in-hospital mortality and U/A level by sex.

\begin{tabular}{|c|c|c|c|c|c|c|}
\hline \multirow{2}{*}{$\begin{array}{l}\text { In-hospital } \\
\text { Mortality }\end{array}$} & \multicolumn{2}{|l|}{ male } & \multicolumn{2}{|l|}{ Female } & \multicolumn{2}{|c|}{ Sex*U/A interaction } \\
\hline & $\mathrm{HR}(95 \% \mathrm{CI})$ & $\mathrm{P}$ & $\operatorname{HR}(95 \% \mathrm{CI})$ & $\mathrm{P}$ & $\beta$ & $\mathrm{P}$ \\
\hline \multirow[t]{2}{*}{ urea/albumin } & $0.520 \square 0.232-$ & 0.112 & $9.380 \square 2.248-$ & 0.002 & 4.290 & 0.004 \\
\hline & $1.165 \square$ & & $39.138 \square$ & & & \\
\hline
\end{tabular}

2 Adjusted for age, Mechanical ventilation, CRRT, vasopressor use, ALT, AST, Creatinine.

3 CRRT: continuous renal replacement treatment; ALT: alanine aminotransferase; AST: aspartate

4 aminotransferase; HR: hazard ratio; CI: confidence interval.

5

6

7

8

9

10

11

12

13

14

15 
1 Figure 1: study algorithm, including patient enrollment and outcomes.

2 Note: low group: U/A $\leq 0.2555$; high group: U/A > 0.2555; SP: severe pneumonia; ROC: receiver 3 operating characteristics curve.

4

5 Figure 2: ROC curve for predicting mortality in patients with SP.

6 U/A had a modest power for predicting in-hospital mortality as suggested by AUC of 0.63 (95\%CI:

$7 \quad 0.55-0.70, P=0.001$ ), with a sensitivity of $84.2 \%$ and a specificity of $37.8 \%$ at a cutoff of 0.2555 .

8

9 Figure 3: Kaplan-Meier survival curve according to U/A level.

10 Compare to the lower group $(\mathrm{U} / \mathrm{A} \leq 0.2555)$, patients in the higher group $(\mathrm{U} / \mathrm{A}>0.2555)$ showed 11 elevated in-hospital mortality. 
Figures

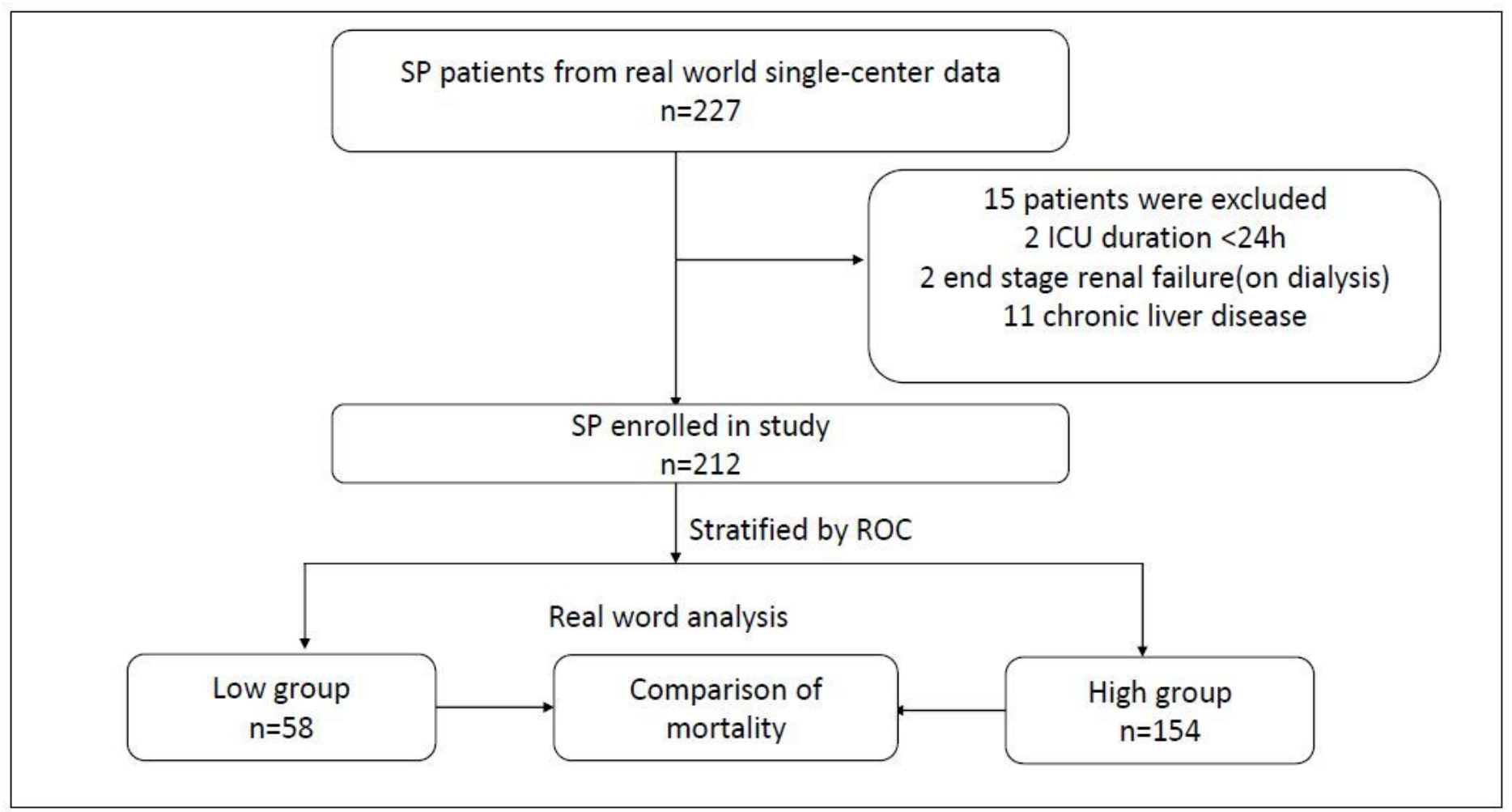

\section{Figure 1}

study algorithm, including patient enrollment and outcomes. Note: low group: $U / A \leq 0.2555$; high group: U/A > 0.2555; SP: severe pneumonia; ROC: receiver operating characteristics curve. 


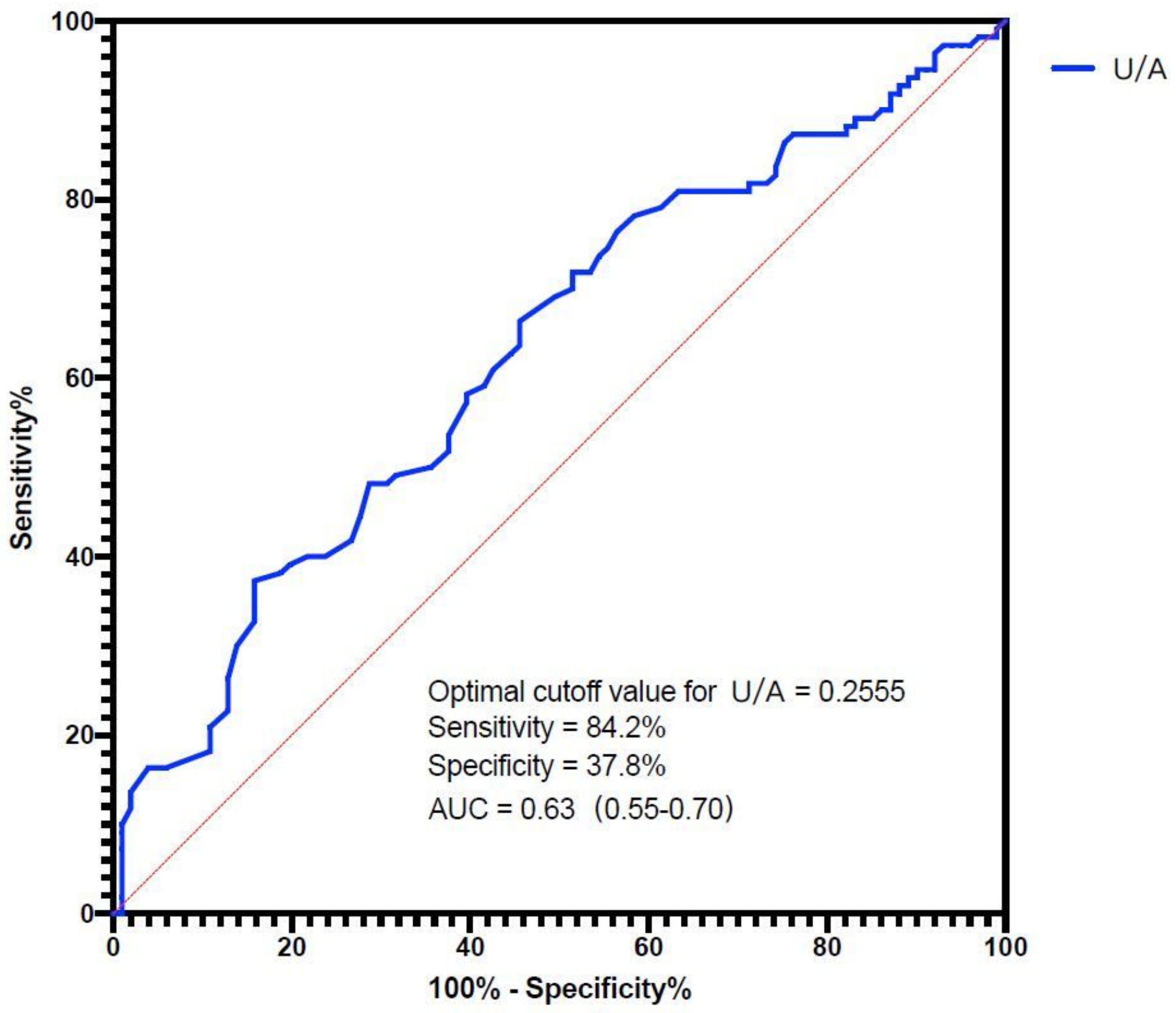

Figure 2

ROC curve for predicting mortality in patients with SP. U/A had a modest power for predicting in-hospital mortality as suggested by AUC of $0.63(95 \% \mathrm{Cl}: 0.55-0.70, \mathrm{P}=0.001)$, with a sensitivity of $84.2 \%$ and a specificity of $37.8 \%$ at a cutoff of 0.2555 . 

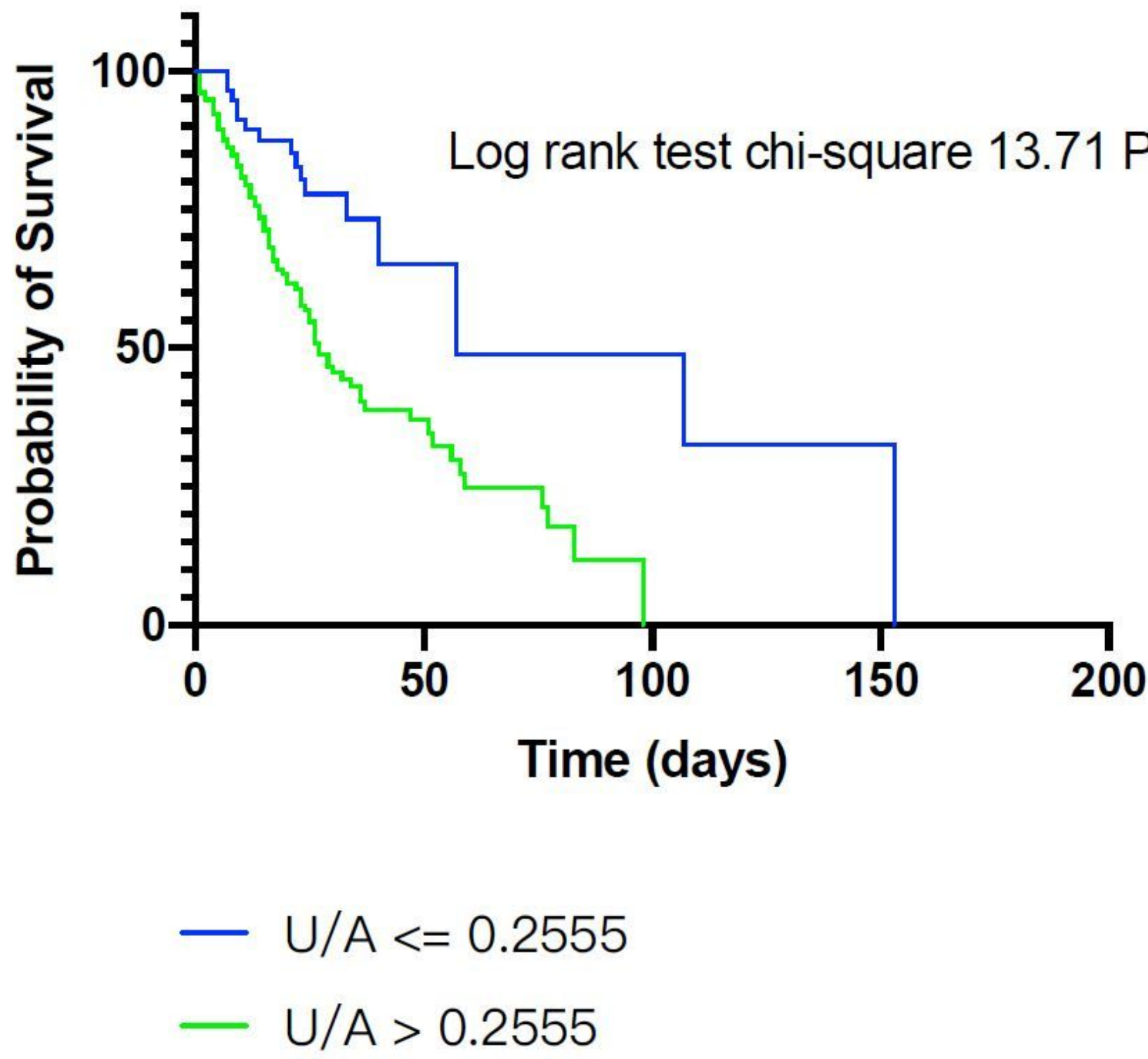

Figure 3

Kaplan-Meier survival curve according to U/A level. Compare to the lower group (U/A $\leq 0.2555$ ), patients in the higher group $(U / A>0.2555)$ showed elevated in-hospital mortality. 